\title{
COEFFICIENT IDEALS
}

\author{
KISHOR SHAH
}

\begin{abstract}
Let $R$ be a $d$-dimensional Noetherian quasi-unmixed local ring with maximal ideal $M$ and an $M$-primary ideal $I$ with integral closure $\bar{I}$. We prove that there exist unique largest ideals $I_{k}$ for $1 \leq k \leq d$ lying between $I$ and $\bar{I}$ such that the first $k+1$ Hilbert coefficients of $I$ and $I_{k}$ coincide. These coefficient ideals clarify some classical results related to $\bar{I}$. We determine their structure and immediately apply the structure theorem to study the associated primes of the associated graded ring of $I$.
\end{abstract}

Let $R$ be a commutative, associative, Noetherian local ring containing an identity. Let $M$ be the maximal ideal of $R$ and $d$ be the Krull dimension of $R$. Let $I$ be an $M$-primary ideal of $R$. Let $\bar{I}$ be the integral closure of $I$ (see [Li1] for an exposition). If $I$ contains a regular element then we let $I^{*}$ be the Ratliff-Rush ideal of $I$ (see [RR, Theorem 2.1]). Thus $I^{*}$ is the unique largest ideal containing $I$ with the property that $I^{* n}=I^{n}$ for all large $n$. In this way, with an ideal $I$ two fairly well-known ideals $\bar{I}$ and $I^{*}$ are associated. Let us first describe them in terms of the Hilbert coefficients of $I$. Recall the classical result of Samuel [Sa, Theorem 9] that the length of $R / I^{n}$ is a polynomial in $n$ for all large values of $n$ of degree $d$. Call this polynomial the Hilbert polynomial of $I$ and write it in the form:

$$
e_{0}(I) \cdot\left(\begin{array}{c}
n+d-1 \\
d
\end{array}\right)+\cdots(-1)^{i} e_{i}(I) \cdot\left(\begin{array}{c}
n+d-i-1 \\
d-i
\end{array}\right) \cdots+(-1)^{d} e_{d}(I)
$$

where $e_{i}(I)$ are the Hilbert coefficients of $I$. Also, $e_{0}(I)$ is called the multiplicity of $I$. Now we describe $\bar{I}$ and $I^{*}$ in terms of the Hilbert coefficients. If $R$ is quasi-unmixed then the integral closure $\bar{I}$ of $I$ may be characterized as the unique largest ideal $I^{\prime}$ containing $I$ for which $e_{0}(I)=e_{0}\left(I^{\prime}\right)$ by [NR, Theorem 1 and R1, Theorem 2]. On the other hand, the Ratliff-Rush ideal $I^{*}$ of $I$ may be characterized as the unique largest ideal $L$ containing $I$ for which $e_{i}(I)=e_{i}(L)$ for all $i$ by [RR, Theorem 2.1].

We prove the existence of a unique chain of ideals, dubbed the coefficient ideals of $I$, between $I$ and $\bar{I}$. These ideals provide a concrete link between the different looking results of Northcott-Rees, Rees, and Ratliff-Rush referred to above. We find their structure and apply the structure theorem to describe

Received by the editors August 23, 1989. Presented to the Society, March 26, 1988.

1980 Mathematics Subject Classification (1985 Revision). Primary 13H15; Secondary 13A15. 
various aspects of $\bar{I}$ and the associated primes of $\operatorname{gr}_{I} R$. We spell out all the main results now.

Theorem 1 (Coefficient ideals $I_{k}$ ). Let $R$ be a quasi-unmixed local ring with maximal ideal $M$. Assume that $R / M$ is infinite and $\operatorname{dim} R=d \geq 1$. Let $I$ be an $M$-primary ideal. Then there exist unique largest ideals $I_{k}$ for $1 \leq k \leq d$, containing $I$ such that

(a) $e_{i}(I)=e_{i}\left(I_{k}\right)$ for $0 \leq i \leq k$.

(b) $I \subset I_{d} \subset \cdots \subset I_{1} \subset \bar{I}=$ integral closure of $I$.

We shall call $I_{k}$ the $k$ th coefficient ideal of $I$.

Indeed $I_{d}$ is the Ratliff-Rush ideal $I^{*}$ if $I$ contains a regular element. We determine the structure of each of the coefficient ideals. The integral closure of $I$ is well known to be an intersection of certain valuation ideals; whereas $I^{*}$ is a union of certain residual quotients of powers of $I$. The dice fall in favor of unions in the following structure theorem for the coefficient ideals of $I$.

Theorem 2 (Structure theorem for the coefficient ideals $I_{k}$ ). In the setup of Theorem 1,

$$
I_{k}=\bigcup\left(I^{N+1}: x_{1}, \ldots, x_{k}\right) \text { for } 1 \leq k \leq d,
$$

for all $N \geq 1$, and all $\underline{x}=x_{1}, \ldots, x_{k}$ extendable to some minimal reduction of $I^{N}$.

It is not at all obvious that the right-hand side in the above equality is even an ideal. Furthermore, one would certainly want to replace the arbitrary union by a single residual quotient. As a matter of fact, a finer structure theorem may be given as follows:

Theorem 3 (Finer structure theorem for the coefficient ideals $I_{k}$ ). In the setup of Theorem 1 ,

$$
I_{k}=\left(I^{N+1}: x_{1}, \ldots, x_{k}\right) \text { for } 1 \leq k \leq d,
$$

for some fixed integer $N \geq 1$ and some fixed minimal reduction $x_{1}, \ldots, x_{d}$ of $I^{N}$.

If $R$ is a normal domain and $I$ is a normal ideal, then the associated graded ring $\operatorname{~gr}_{I} R$ has only minimal associated primes. It is natural to ask how the associated primes of $\mathrm{gr}_{I} R$ behave when $I^{s}=\left(I^{s}\right)_{k} \forall s$. Fulton expresses a need to obtain a criterion to identify the irreducible components of $\operatorname{gr}_{I} R$ with their multiplicities in [Fu, p. 13]. We obtain a result which spells out how the coefficient ideals control the associated primes of $\mathrm{gr}_{I} R$.

Theorem 4 (Coefficient ideals $I_{k}$ and associated primes of $\operatorname{gr}_{I} R$ ). Fix the setup of Theorem 1. Fix a $k$ with $1 \leq k \leq d$. Then, $I^{S}=\left(I^{s}\right)_{k}$ for every $s$ if and only if ht. $P<k$ for every associated prime $P$ of $\mathrm{gr}_{I} R$.

Our final theorem of this paper relates the statement $I^{S}=\left(I^{s}\right)_{k} \forall s$ to the presence of regular sequences in $\operatorname{gr}_{I} R$. 
Theorem 5 (Grade of $\operatorname{gr}_{I} R$ and $\left.I^{s}=\left(I^{s}\right)_{k}\right)$. Fix the setup of Theorem 1. Let $N_{1}=$ irrelevant maximal ideal of $\operatorname{gr}_{I} R$. Fix $a k$ with $1 \leq k \leq d$. Then, grade $N_{1} \geq k$ implies that $I^{s}=\left(I^{s}\right)_{j}$ for $d+1-k \leq j \leq d$, and for all $s \geq 1$.

Our general references for this paper are [Ma, Mc]. We shall presuppose elementary knowledge of reductions [Li1, NR], of system of parameters [Ho], and of Rees valuations [Mc, R2]. For the convenience of the quick-browse reader the statements of all the main theorems have already been recorded in the Introduction. To keep the paper short the easier proofs will be left as exercises to the reader. Nevertheless we note that $[\mathrm{S}]$ contains a detailed account of this paper.

\section{EXISTENCE AND UNIQUENESS OF COEFFICIENT IDEALS}

In this section we prove Theorem 1 stated in the Introduction. We begin by stating and proving two remarks in a general setting. These remarks are essentially used in the proof of Theorem 1. While we use standard notation, note that

(a) A polynomial of degree -1 is the zero polynomial.

(b) If $N$ is an $R$-module then $l(N)$ denotes the length of $N$.

Remark 1(A). Let $R$ be a Noetherian local ring with maximal ideal $M$ and $\operatorname{dim} R=d \geq 1$. Suppose $I \subset J$ are $M$-primary. Fix $k$ with $1 \leq k \leq d$. Then for all large $n$, we have $e_{i}(I)=e_{i}(J)$ with $0 \leq i \leq k$ iff $l\left(J^{n} / I^{n}\right) \leq P(n)$, where $P(n)$ is some polynomial in $n$ of degree at most $d-(k+1)$.

Proof. We need only observe that, for large $n$,

$$
l\left(J^{n} / I^{n}\right)=l\left(R / I^{n}\right)-l\left(R / J^{n}\right)=\sum_{i=0}^{i=d}(-1)^{i}\left[e_{i}(I)-e_{i}(J)\right] \cdot\left(\begin{array}{c}
n+d-i-1 \\
d-i
\end{array}\right) .
$$

Remark 1(B). Let $R$ be a Noetherian local ring with maximal ideal $M$ and $\operatorname{dim} R=d \geq 1$. Suppose that $I \subset I^{\prime} \subset J$ are $M$-primary ideals. Fix $k$ with $1 \leq k \leq d$. Then $e_{i}(I)=e_{i}(J)$ with $0 \leq i \leq k$ iff $e_{i}(I)=e_{i}\left(I^{\prime}\right)=e_{i}(J)$ with $0 \leq i \leq k$.

Proof. We need only observe that $l\left(I^{\prime n} / I^{n}\right) \leq l\left(J^{n} / I^{n}\right)$ and that we may simply apply Remark 1(A) to obtain the conclusion of Remark 1(B).

Proof of Theorem 1. Fix $k$ with $1 \leq k \leq d$. Consider the set $V$ of all ideals $L$ with the property that $I \subset L$ and $e_{i}(I)=e_{i}(L)$ for $0 \leq i \leq k$. Now $V$ is nonempty as $I$ belongs to $V$. So $V$ contains a maximal element $J$, say. We shall prove that $J$ is the unique maximal element in $V$. Suppose $L$ belongs to $V$. We prove $L \subset J$. Pick any $x \in L$. Now $I \subset(I, x) \subset L$. So $e_{i}(I)=e_{i}(I, x)=e_{i}(L)$ for $0 \leq i \leq k$ follows from Remark $1(\mathrm{~B})$. In particular, $e_{0}(I)=e_{0}(I, x)$. By [R1, Theorem 3.2], $(I, x)^{t+1}=(I, x)^{t} I$ holds for some $t \geq 0$. In particular, $x^{t+1} \in(I, x)^{t} I$. Hence $x^{t+1} \in(J, x)^{t} J$. This implies 
that $(J, x)^{t+1}=(J, x)^{t} J$ also holds. Hence $(J, x)^{n}=(J, x)^{t} J^{n-t}$ holds for a fixed $t$ and all $n \geq t$. We obtain

$$
\begin{aligned}
l\left[(J, x)^{n} / J^{n}\right] & =l\left[(J, x)^{t} J^{n-t} / J^{n}\right] \leq \sum_{i=1}^{i=t} l\left[J^{n-i} x^{i}+J^{n} / J^{n}\right] \\
& \leq \sum_{i=1}^{i=t} l\left[J^{n-i} x^{i}+J^{n} / I^{n}\right] \leq \sum_{i=1}^{i=t}\left(l\left[J^{n-i} x^{i}+I^{n} / I^{n}\right]+l\left[J^{n} / I^{n}\right]\right) \\
& \leq \sum_{i=1}^{i=t}\left(l\left[J^{n-i} x^{i}+I^{n} / I^{n-i} x^{i}+I^{n}\right]+l\left[I^{n-i} x^{i}+I^{n} / I^{n}\right]+l\left[J^{n} / I^{n}\right]\right) \\
& \leq \sum_{i=1}^{i=t}\left(l\left[J^{n-i} / I^{n-i}\right]+l\left[(I, x)^{n} / I^{n}\right]+l\left[J^{n} / I^{n}\right]\right) .
\end{aligned}
$$

But $e_{i}(I)=e_{i}(I, x)$ and $e_{i}(I)=e_{i}(J)$ holds for $0 \leq i \leq k$. We apply Remark 1 (A) to every term on the right-hand side of the last inequality to obtain that $l\left[(J, x)^{n} / J^{n}\right]$ is bounded by a polynomial in $n$ for all large $n$ of degree at most $d-(k+1)$. This shows, again by Remark $1(\mathrm{~A})$, that $e_{i}(J)=e_{i}(J, x)$ holds for $0 \leq i \leq k$. As $J$ was maximal in $V$, we deduce that $x \in J$. So $L \subset J$. Thus $J$ is the unique maximal element in $V$. Finally set $J=I_{k}$. This proves (a).

Since $e_{0}(I)=e_{0}\left(I_{k}\right)$ and $I \subset I_{k}$ it follows that $I_{k} \subset \bar{I}$. Since $I_{k}$ is the unique largest ideal containing $I$ and satisfying (a) it follows that $I_{t} \subset I_{k}$ if $1 \leq k \leq t \leq d$. This proves (b). This also completes the proof of Theorem 1 .

Corollary 1(C). Fix the setup of Theorem 1 .

$$
I \subset J \subset I_{k} \subset \bar{I} \text { iff } I \subset J \text { and } e_{i}(I)=e_{i}(J) \quad \forall 0 \leq i \leq k .
$$

Remark 1(D). The above corollary clarifies further the classical result [NR, Theorem 1 and R1, Theorem 3.2] that $I$ is a reduction of $J$ iff $e_{0}(I)=e_{0}(J)$.

Corollary $1(\mathbf{E})$. Fix the setup of Theorem 1 with I containing a regular element. Consider the dth coefficient ideal $I_{d}$ and the Ratliff-Rush ideal $I^{*}$ of $I$. We have $I_{d}=I^{*}$.

Remark $1(\mathrm{~F})$. Fix the setup of Theorem 1 . Assume that $I$ is integrally closed. Then $I=I_{d}=\cdots=I_{1}=\bar{I}$.

\section{Algebraic STRUCTURE OF THE COEFFICIENT IDEALS}

In this section we prove Theorem 2 stated in the Introduction. This theorem uncovers the algebraic structure of the coefficient ideals. We require six lemmas to put together a proof.

Lemma 2(A) allows us to view minimal reductions of $I^{N}$ as systems of parameters in $\mathrm{gr}_{I} R$ instead of in $\operatorname{gr}_{I} R \otimes R / M$. Lemma 2(B) essentially says that a certain Rees valuation remains constant over reduction elements. To understand the arguments in the proof, elementary knowledge of Rees valuations is required: we give a very brief summary of it later. Lemma $2(C)$ is a 
necessary technical statement. The next three lemmas (2(D), 2(E), 2(F)) are essentially prime avoidance results whose proofs are left as easy exercises to the pencil-reader. However, armchair-readers will find the proofs in [S, Chapter 1].

Lemma 2(A) (Reductions of ideals and $\operatorname{gr}_{I} R$ (also see [GHO])). Let $R$ be a local ring with maximal ideal $M$. Let $I$ be an M-primary ideal. Let $x_{1}, \ldots, x_{s} \in I^{N}$ for some fixed $N \geq 1$, and some $s \geq 1$ with $x_{1}^{\prime}, \ldots, x_{s}^{\prime}$ denoting their images in $I^{N} / I^{N+1}$. Then

$$
l\left(\operatorname{gr}_{I} R /\left(x_{1}^{\prime}, \ldots, x_{s}^{\prime}\right) \operatorname{gr}_{I} R\right)<\infty \text { iff } x_{1}, \ldots, x_{s} \text { form a reduction of } I^{N} .
$$

Proof. Suppose $l\left(\mathrm{gr}_{I} /\left(x_{1}^{\prime}, \ldots, x_{s}^{\prime}\right) \operatorname{gr}_{I} R\right)<\infty$. Then for some large $n$,

$$
\left(x_{1}^{\prime}, \ldots, x_{s}^{\prime}\right)\left(I^{N n} / I^{N n+1}\right)=I^{N+N n} / I^{N+N n+1} \text {. }
$$

Hence

$$
\left(x_{1}^{\prime}, \ldots, x_{s}^{\prime}\right) I^{N n}+I^{N+N n+1}=I^{N+N n} .
$$

Nakayama's lemma gives $\left(x_{1}, \ldots, x_{s}\right) I^{N n}=I^{N+N n}$. This means that $x_{1}, \ldots$, $x_{s}$ form a reduction of $I^{N}$. Suppose next that $x_{1}, \ldots, x_{s}$ form a reduction of $I^{N}$. Then for some $n \geq 1$, we have $\left(x_{1}, \ldots, x_{s}\right) I^{N n}=I^{N+N n}$. Thus

$$
\left(x_{1}^{\prime}, \ldots, x_{s}^{\prime}\right)\left(I^{N n} / I^{N n+1}\right)=I^{N+N n} / I^{N+N n+1} .
$$

This implies that $l\left(\operatorname{gr}_{I} R /\left(x_{1}^{\prime}, \ldots, x_{s}^{\prime}\right) \operatorname{gr}_{I} R\right)<\infty$.

Summary of Rees valuations. Suppose that $R$ is an analytically unramified local domain with $\operatorname{dim} R \geq 1$. Let $I$ be any nonzero ideal in $R$. Let $t$ be an indeterminate. Set $T=\bigoplus I^{s} t^{s}$, where $s$ ranges over the integers but $I^{s}=R$ if $s \leq 0$. Set $\bar{T}=\bigoplus \overline{I^{s}} t^{s}$, where $s$ ranges over the integers but $\overline{I^{s}}=R$ if $s \leq 0$. Let $N$ and $N^{\prime}$ be the unique maximal irrelevant ideals of $T$ and $\bar{T}$ respectively. Then the following well-known statements constitute a brief summary of the theory of Rees valuations. Some general references are [Mc, R2].

(V.1) $\bar{T}$ is integrally closed in $R[t, u]$ where $u=t^{-1}$.

(V.2) $\bar{T}[t]=R[t, u]$.

(V.3) $\bar{T}$ is a Noetherian domain.

(V.4) $\operatorname{dim} T=\operatorname{dim} \bar{T}=d+1 \geq 2$.

(V.5) $u \bar{T}$ is an integrally closed ideal in $\bar{T}$.

(V.6) If $P_{1}, \ldots, P_{r}$ are all the associated primes of $u \bar{T}$ in $\bar{T}$, then $(\bar{T})_{P_{1}}$, $\ldots,(\bar{T})_{P_{r}}$ are all discrete valuation rings.

(V.7) $P_{1}, \ldots, P_{r}$ naturally induce discrete valuations $V_{1}, \ldots, V_{r}$ on the fraction field of $\bar{T}$.

(V.8) Set $V_{I}(x)=n$ if $x \in I^{n}-I^{n+1}$. Set $V_{I}(0)=\infty$. Set $\bar{V}_{I}(x)=$ limit $V_{I}\left(x^{n}\right) / n$ as $n \rightarrow \infty$. Then $\bar{V}_{I}(x) \geq k$ if and only if $x \in \overline{I^{k}}$.

(V.9) $\bar{V}_{I}(x)=\min \left\{V_{j}(x) / V_{j}(I) \mid j=1, \ldots, r\right\}$.

(V.10) $\bar{V}_{I}(x)=n \bar{V}_{I^{n}}(x) \forall n \geq 1$. 
Lemma 2(B) (Rees valuations and minimal reductions). Let $R$ be an analytically unramified, universally catenary local domain with maximal ideal $M$. Let $\operatorname{dim} R=d \geq 1$. Let $I$ be an $M$-primary ideal. Let $x_{1}, \ldots, x_{s}$ form a minimal reduction of $I$. Then $V\left(x_{i}\right)=V(I)$ for every Rees valuation $V$ of $I$ with $i=1, \ldots, d$.

Proof. We shall freely use the notations and facts recorded earlier in the summary of Rees valuations. Since $\mathrm{gr}_{I} R \simeq T / u T$, the hypothesis on $x_{1}, \ldots, x_{d}$ implies that $N$ is primary to $\left(x_{1} t, \ldots, x_{d} t, u\right) T$. It follows that

$$
\text { ht. }\left(x_{1} t, \ldots, x_{d} t, u\right) T=d+1 \text {. }
$$

We claim that ht. $\left(x_{1} t, \ldots, x_{d} t, u\right) \bar{T}=d+1$. To see this, let $Q$ be a prime minimal over $\left(x_{1} t, \ldots, x_{d} t, u\right) \bar{T}$. Since $\bar{T}$ is finitely generated over a universally catenary domain $T$, we can apply the dimension formula: ht. $Q=$ ht. $Q \cap T+a-b$, where $a=\operatorname{tr}$. deg. of $\bar{T}$ over $T$, and $b=\operatorname{tr}$. deg. of $\bar{T} / Q$ over $T / Q \cap T$. However $a=0$ because $T, \bar{T}$ have the same quotient field. Next $b=0$ because $\bar{T} / Q$ is integral over $T / Q \cap T$. So ht. $Q=$ ht. $Q \cap T$. As $Q \cap T=$ $N$, it follows that ht. $Q=\operatorname{dim} . T_{N}=d+1$. Thus ht. $\left(x_{1} t, \ldots, x_{d} t, u\right) \bar{T}=d+1$ as claimed.

We next claim that ht. $\left(x_{i} t, u\right)=2$. To see this, we observe that $x_{1} t, \ldots$, $x_{d} t, u$ form a system of parameters of the catenary local domain $(\bar{T})_{N}$. It follows that ht. $\left(x_{i} t, u\right)(\bar{T})_{N}=2$. Because $\left(x_{i} t, u\right) \bar{T}$ is a graded ideal, we get that ht. $\left(x_{i} t, u\right) \bar{T}=2$ as claimed.

We now claim that $V\left(x_{i}\right)=V(u)$. It is enough to show that $x_{i} t \notin$ any height 1 prime $P$ in $\bar{T}$ which also contains $u$. This is true because we have shown ht. $\left(x_{i} t, u\right) \bar{T}=2$. Thus $V\left(x_{i} t\right)=0$ giving $V\left(x_{i}\right)=V(u)$ as claimed.

We finally claim that $V(I)=V(u)$. To see this, suppose $P$ is a height 1 prime in $\bar{T}$ which also contains $u$ and $(I t) \bar{T}$. So $P \supset\left(x_{1} t, \ldots, x_{d} t, u\right) \bar{T}$. It follows that $P=N^{\prime}$, giving $\operatorname{dim} . \bar{T}=\operatorname{dim} \cdot(\bar{T})_{P}=1$. This is a contradiction, as $\operatorname{dim} . \bar{T}=d+1 \geq 2$. It follows that $V(I t)=0$, giving $V(I)=V(u)$ as claimed.

Our punch line is: $V\left(x_{i}\right)=V(u)=V(I)$. This proves the lemma.

Lemma $2(C)$ (Killing of a reduction element). Let $R$ be a quasi-unmixed local ring with maximal ideal $M$. Let $\operatorname{dim} . R=d \geq 1$. Let $I$ be an $M$-primary ideal. Let $x_{1}, \ldots, x_{d}$ form a minimal reduction of $I^{N}$ for some fixed $N \geq 1$. Suppose $y x_{i} \in I^{N+1}$ for some $y \in R$. Then $y \in \bar{I}$.

Proof. We may assume that $R$ is complete. To see this let ${ }^{-}$denote the natural map from $R$ to its completion $\widehat{R}$. We note that $\hat{x}_{1}, \ldots, \hat{x}_{d}$ form a minimal reduction of $\left(\widehat{I}^{N}\right.$. Suppose the conclusion of the lemma holds for $\widehat{R}$. Then $y \in \overline{(\widehat{I})^{N}} \cap R=\bar{I}$, by [Li2, p. 792]. Thus we may assume that $R$ is complete.

We may further assume that $R$ is a domain. To see this, let $P_{1}, \ldots, P_{t}$ be all the minimal primes of $R$. Since $R$ is quasi-unmixed, $\operatorname{dim} . R / P_{i}=d$ for $i=1, \ldots t$. So the images of $x_{1}, \ldots, x_{d}$ form a minimal reduction of 
$\left(I+P_{i}\right) / P_{i}$. Suppose the conclusion of the lemma holds for $R / P_{i}$ for $i=$ $1 \ldots, t$. Then the image of $y$ in $R / P_{i}$ will belong to $\overline{\left(I+P_{i}\right) / P_{i}}$ for $i=$ $1, \ldots, d$. This will imply that $y \in \bar{I}$. Thus we may assume that $R$ is a complete domain. Observe that $R$ is an analytically unramified, universally catenary domain. Hence $V\left(I^{N}\right)=V\left(x_{i}\right)$ for every Rees valuation $V$ of $I^{N}$ by Lemma 2(B). So $y x_{i} \in I^{N+1}$ gives $V(y)+V\left(x_{i}\right) \geq V\left(I^{N}\right)+V(I)$. Hence $V(y) \geq V(I)$. So $V(y) \geq \frac{1}{N} V\left(I^{N}\right)$. Thus $\bar{V}_{I^{N}}(y) \geq \frac{1}{N}$ follows from (V.9). So $\bar{V}_{I}(u) \geq 1$ follows from (V.10). Finally $y \in \bar{I}$ follows from (V.8). This proves the lemma.

Lemma 2(D) (Obviously a folklore prime avoidance). Let $R$ be a nonnegatively graded Noetherian ring. Set $R=\bigoplus_{0}^{\infty} R_{n}$. Suppose that $R_{0}$ is an Artinian local ring. Let $N$ be the unique homogeneous maximal ideal of $R$. Suppose that $\operatorname{dim} . R=$ ht. $N=d \geq 1$. Then we may choose homogeneous elements $a_{1}, \ldots, a_{d}$ of positive degree such that

(1) $a_{1}, \ldots, a_{d}$ are homogeneous elements of equal degree,

(2) $l\left(R /\left(a_{1}, \ldots, a_{d}\right)\right)<\infty$

(3) $\operatorname{dim} . R /\left(a_{1}, \ldots, a_{j}\right)=\operatorname{dim} . R-j$ for $1 \leq j \leq d$.

Lemma 2(E) (An obvious generalization of folklore prime avoidance [S, p. 23]). Let $R$ be a nonnegatively graded ring. Set $R=\bigoplus_{0}^{\infty} R_{n}$. Suppose that $R_{0}$ is an Artinian local ring. Let $N$ be the unique homogeneous maximal ideal of $R$. Suppose that $\operatorname{dim} . R=\mathrm{ht} . N=d \geq 1$. Let $A$ be a homogeneous ideal of $R$ such that $\operatorname{dim} . R / A \leq d-k$. Then we may choose homogeneous elements $a_{1}, \ldots a_{d}$ of $R$ such that

(1) $a_{1}, \ldots, a_{d}$ are homogeneous elements of equal degree,

(2) $l\left(R /\left(a_{1}, \ldots, a_{d}\right)\right)<\infty$

(3) $\operatorname{dim} . R /\left(a_{1}, \ldots, a_{j}\right)=\operatorname{dim} . R-j$ for $1 \leq j \leq d$,

(4) If $k \geq 1$ then $a_{1}, \ldots a_{k}$ lie in $A$.

Lemma 2(F) (Refined generalized prime avoidance lemma [S, p. 27]). Let $R$ be a nonnegatively graded Noetherian ring. Set $R=\bigoplus_{0}^{\infty} R_{n}$. Suppose that $R_{0}$ is an Artinian local ring. Let $N$ be the unique homogeneous maximal ideal of $R$. Suppose that $\operatorname{dim} . R=\mathrm{ht} . N=d \geq 1$. Let $A_{k}$ be homogeneous ideals of $R$ such that $\operatorname{dim} . R / A_{k} \leq d-k$ for $1 \leq k \leq d$. Furthermore suppose $A_{1} \subset \cdots \subset A_{d}$. Then we may always pick homogeneous elements $a_{1}, \ldots, a_{d}$ of $R$ enjoying all the following properties:

(1) $a_{1}, \ldots, a_{d}$ are homogeneous elements of equal degree,

(2) $l\left(R /\left(a_{1}, \ldots, a_{d}\right)\right)<\infty$,

(3) $\operatorname{dim} . R /\left(a_{1}, \ldots, a_{k}\right)=\operatorname{dim} . R-k$ for $1 \leq k \leq d$,

(4) $a_{1}, \ldots, a_{k}$ all lie in $A_{k}$ for $1 \leq k \leq d$.

Proof of Theorem 2. First we show that $I_{k} \subset$ right-hand side for a fixed $k$. Suppose $y \in I_{k}$. Then $I \subset(I, y)$ and $e_{i}(I)=e_{i}\left(I_{k}\right)$ for $0 \leq i \leq k$, by 
the definition of $I_{k}$. So $l\left[(I, y)^{n} / I^{n}\right] \leq P(n)$ for all large $n$, where $P(n)$ is a polynomial in $n$ of degree $d-(k+1)$ by Remark 1(A). In particular, $l\left[(I, y) I^{n-1} / I^{n}\right] \leq P(n)$. Set

$$
E=((I, y) / I) \mathrm{gr}_{I} R=\bigoplus_{1}^{\infty}(I, y) I^{n-1} / I^{n} .
$$

Now $E$ is nothing but the graded $\mathrm{gr}_{I} R$-submodule of $\mathrm{gr}_{I} R$ generated by $(I, y) / I$. Set $A_{k}=$ annihilator of $E$ in $\mathrm{gr}_{I} R$. So $E$ has module dimension at most $d-k$. Thus $\operatorname{gr}_{I} R / A_{k}$ has Krull dimension at most $d-k$. Furthermore $A_{k}$ is a homogeneous ideal. Pick homogeneous elements $x_{1}^{\prime}, \ldots, x_{d}^{\prime}$ in $\operatorname{gr}_{I} R$ as in Lemma 2(E) such that

(1) $x_{1}^{\prime}, \ldots, x_{d}^{\prime}$ are homogeneous of degree $N \geq 1$,

(2) $l\left(\operatorname{gr}_{I} R /\left(x_{1}^{\prime}, \ldots, x_{d}^{\prime}\right)\right)<\infty$,

(3) $\operatorname{dim} .\left(\operatorname{gr}_{I} R /\left(x_{1}^{\prime}, \ldots, x_{k}^{\prime}\right)\right)=d-k$,

(4) $x_{1}^{\prime}, \ldots, x_{k}^{\prime}$ all lie in $A_{k}$.

Let $x_{1}, \ldots, x_{d}$ be any preimages of $x_{1}^{\prime}, \ldots, x_{d}^{\prime}$ in $I^{N}$. Then $x_{1}, \ldots, x_{d}$ form a minimal reduction of $I^{N}$ by Lemma 2(A) and (2) just above. Since $x_{1}^{\prime}, \ldots, x_{k}^{\prime}$ lie in $A_{k}$ we obtain that $y \in\left(I^{N+1}: x_{1}, \ldots, x_{k}\right)$. Thus $I_{k} \subset$ right-hand side as needed to be shown.

Second, we shall show that $I_{k} \supset$ right-hand side. Suppose $y \in\left(I^{N+1}: x_{1}\right.$, $\left.\ldots, x_{k}\right)$. In particular $y x_{1} \in I^{N+1}$. Thus $y \in \bar{I}$ follows from Lemma $2(\mathrm{C})$. Hence $I$ is a reduction of $(I, y)$. This means that $(I, y)^{s+n}=(I, y)^{s} I^{n}$ for some fixed $s$ and all $n \geq 1$. We now carry out a computation:

$$
\begin{aligned}
l\left[(I, y)^{s+n} / I^{s+n}\right] & =l\left[(I, y)^{s} I^{n} / I^{s+n}\right] \\
& =\sum_{i=1}^{i=s} l\left[(I, y)^{i} I^{n+s-i} /(I, y)^{i-1} I^{n+s-i+1}\right] \\
& =\sum_{i=1}^{i=s} l\left[(I, y)^{i-1} I^{s-i}(I, y) I^{n} /(I, y)^{i-1} I^{s-i} I^{n+1}\right] \\
& \leq \sum_{i=1}^{i=s} c_{i} l\left[(I, y) I^{n} / I^{n+1}\right]
\end{aligned}
$$

for some positive constants $c_{i}$. The latter inequality can be seen by canonically mapping for each $i$

$$
\bigoplus_{1}^{c_{i}}(I, y) I^{n} / I^{n+1} \rightarrow(I, y)^{i-1} I^{s-i}(I, y) I^{n} /(I, y)^{i-1} I^{s-i} I^{n+1} \rightarrow 0 .
$$

We may choose $c_{i}$ to be the minimal number of generators of $(I, y)^{i-1} I^{s-i}$. Set $c=\sum c_{i}$. Thus

$$
l\left[(I, y)^{s+n} / I^{s+n}\right] \leq c \cdot l\left[(I, y) I^{n} / I^{n+1}\right] .
$$


But $l\left[(I, y) I^{n} / I^{n+1}\right]$ is a polynomial in $n$, for large $n$, whose degree equals $\operatorname{dim} . M-1$ where $M=((I, y) / I) \operatorname{gr}_{I} R$. In fact, $M$ is nothing but the graded $\mathrm{gr}_{I} R$-submodule of $\operatorname{gr}_{I} R$ generated by $(I, y) / I$. But $\operatorname{dim} . M=$ Krull dimension of $\operatorname{gr}_{I} R / A$ where $A$ is the annihilator of $M$. Since $y \in\left(I^{n+1}: x_{1}, \ldots, x_{k}\right)$ it follows that $x_{1}^{\prime}, \ldots, x_{k}^{\prime}$ belong to $A$. Thus Krull dimension of $\operatorname{gr}_{I} R / A \leq$ Krull dimension of $\operatorname{gr}_{I} R /\left(x_{1}^{\prime}, \ldots, x_{k}^{\prime}\right)=d-k$. The latter equality is true because $\operatorname{gr}_{I} R$ is equidimensional; whence we may localize at the irrelevant maximal ideal, go modulo a minimal prime, and reduce to the catenary local domain case. Thus $l\left[(I, y)^{s+n} / I^{s+n}\right]$ is bounded by a polynomial in $n$ for all large $n$ of degree $d-(k+1)$. Hence we obtain $e_{i}(I)=e_{i}(I, y)$ with $0 \leq i \leq k$ by Remark 1(A). Therefore $I \subset(I, y) \subset I_{k}$ follows from the definition of $I_{k}$. In particular $y \in I_{k}$. Hence $I_{k} \supset\left(I^{N+1}: x_{1}, \ldots, x_{d}\right)$. This completes the proof of the theorem.

Corollary 2(G). The right-hand side of the equality in the statement of Theorem 2 is in fact an ideal (this is not a priori obvious).

\section{ANOTHER STRUCTURE THEOREM FOR THE COEFFICIENT IDEALS}

In this section we prove Theorem 3 stated in the Introduction. A natural question pertaining to the infinite union in Theorem 2 is whether the union may be replaced by a finite union. By selecting parameters in a refined manner (Lemma 2(F) in §2) we show that $I_{k}$ is a single residual quotient.

Proof of Theorem 3. First we shall show that there is an integer $N \geq 1$ and there is a minimal reduction $x_{1}, \ldots, x_{d}$ such that $I_{k} \subset\left(I^{N+1}: x_{1}, \ldots, x_{k}\right)$ for $1 \leq k \leq d$. Fix $I_{k}$. We know that $I \subset I_{k}$ and $e_{i}(I)=e_{i}\left(I_{k}\right)$ with $0 \leq i \leq k$ by the definition of $I_{k}$. We also know that $l\left[\left(I_{k}\right)^{n} / I^{n}\right] \leq P(n)$ for all large $n$, where $P(n)$ is a polynomial in $n$ of degree $d-(k+1)$. In particular $l\left[I_{k} \cdot I^{n-1} / I^{n}\right] \leq P(n)$. Set $E^{(k)}=\left(I_{k} / I\right) \mathrm{gr}_{I} R$. Set $A_{k}=$ annihilator of $E^{(k)}$ in $\operatorname{gr}_{I} R$. Thus $E^{(k)}$ has module dimension of at most $d-k$. So $\operatorname{gr}_{I} R / A_{k}$ has Krull dimension of at most $d-k$. This holds for all $k$ such that $1 \leq k \leq d$. Further, $A_{1} \subset \cdots \subset A_{d}$. Further, each $A_{k}$ is a homogeneous ideal. By our refined prime avoidance lemma we pick homogeneous elements $x_{1}^{\prime}, \ldots, x_{d}^{\prime}$ in $\operatorname{gr}_{I} R$ of some fixed degree $N \geq 1$ such that $l\left(\mathrm{gr}_{I} R /\left(x_{1}^{\prime}, \ldots, x_{d}^{\prime}\right)\right)<\infty$ and that $x_{1}^{\prime}, \ldots, x_{k}^{\prime}$ lying in $A_{k}$ for $1 \leq k \leq d$. Let $x_{1}, \ldots, x_{d}$ be any preimages of $x_{1}^{\prime}, \ldots, x_{d}^{\prime}$ in $I^{n}$. Then $x_{1}, \ldots, x_{d}$ form a minimal reduction of $I^{n}$ by Lemma 2(A). Of course as $x_{1}^{\prime}, \ldots, x_{k}^{\prime}$ lie in $A_{k}$, we obtain that $I_{k} \subset\left(I^{N+1}: x_{1}, \ldots, x_{k}\right)$ for $1 \leq k \leq d$.

Second, we need only observe that $I_{k} \supset\left(I^{N+1}: x_{1}, \ldots, x_{k}\right)$ for $1 \leq k \leq d$, by a direct appeal to Theorem 2 . This completes the proof of our Theorem 3 .

Corollary 3(A). Consider the ideal $I_{d}$. Fix the notation as in the theorem. Then $I_{d}=\left(I^{N+1}: x_{1}, \ldots, x_{d}\right)$ for some fixed integer $N \geq 1$ and some minimal reduction $x_{1}, \ldots, x_{d}$ of $I^{N}$. 
Remark 3(B). We point out that in spite of Theorem 3 and Corollary 3(A), it is unreasonable to claim in general that $I_{k}=\left(I^{N+1}: x_{1}, \ldots, x_{k}\right)$ for all large $N \geq 1$ and any minimal reduction $x_{1}, \ldots, x_{d}$ of $I^{N}$ : For suppose this claim holds. Let $y \in I_{k}$. Let $z_{1}, \ldots, z_{d}$ be a minimal reduction of $I^{N}$. The arbitrary nature of $z_{1}, \ldots, z_{d}$ implies that if $y \in I_{k}$, then $y z_{i} \in I^{N+1}$ for $1 \leq i \leq d$. So $y \in I_{d}$ follows from Theorem 2. This implies $I_{k} \subset I_{d}$, which will not be the case in general if $k \leq d$.

Corollary 3(C). Fix the setup of Theorem 3. Assume that $I^{n} / I^{n+1}$ is $R / I-$ free for all large $n$. Then $I=I_{d}$.

\section{APPLICATIONS OF THE COEFFICIENT IDEALS}

In this section we prove Theorem 4 and Theorem 5 stated in the Introduction. These results are applications of the coefficient ideals to understand some aspects of $\mathrm{gr}_{I} R$. Theorem 4 explains the nature of associated primes of $\mathrm{gr}_{I} R$ in terms of the coefficient ideals. The proof depends on the fact that associated primes may be expressed as residual quotients and ideals of high height must contain regular elements if all the associated primes have a relatively low height. On the other hand, Theorem 5, which is technical, states the effect of grade in $\operatorname{gr}_{I} R$ on the coefficient ideals.

Proof of Theorem 4. First, assume that $I^{s}=\left(I^{s}\right)_{k}$ for every $s$. Pick any associated prime $P$ of $\operatorname{gr}_{I} R$. Suppose that ht. $P \geq k$. Since $P$ is graded there exists $b \in I^{s-1}-I^{s}$ with $s \geq 1$ such that $P=\left(0^{\prime}: b^{\prime}\right)$, where $b^{\prime}$ is the order image of $b$ in $\operatorname{gr}_{I} R$. We can choose $x_{1}, \ldots, x_{k}$ in $R$ extendable to a minimal reduction of $\left(I^{S}\right)^{N}$ for some $N$ such that their canonical images $x_{1}^{\prime}, \ldots, x_{k}^{\prime}$ in $\operatorname{gr}_{I} R$ lie in $P$. So $b\left(x_{1}, \ldots, x_{k}\right) \subset I^{s-1+s N+1}$, giving $b \in$ $\left(\left(I^{S}\right)^{N+1}: x_{1}, \ldots, x_{k}\right)$. This implies $b \in\left(I^{S}\right)_{k}$ by Theorem 2. So $b \in I^{s}$ which is a contradiction. Hence ht. $P<k$ as required.

Second, assume that ht. $P<k$ for every associated prime $P$ of $\operatorname{gr}_{I} R$. Let $x_{1}, \ldots, x_{d}$ be any minimal reduction of $\left(I^{S}\right)^{N}$ for any $N$. Let $x_{1}^{\prime}, \ldots, x_{d}^{\prime}$ be their canonical images in $\operatorname{gr}_{I} R$. Then ht. $\left(x_{1}^{\prime}, \ldots, x_{d}^{\prime}\right)=d$. Since $\mathrm{gr}_{I} R$ is equidimensional by (a well-known Ratliff result [Ra, p. 121] it follows that ht. $\left(x_{1}^{\prime}, \ldots, x_{k}^{\prime}\right)=k$. So $\left(x_{1}^{\prime}, \ldots, x_{k}^{\prime}\right)$ contains a nonzero divisor. This implies that $\left(\left(I^{s}\right)^{N+1}: x_{1}, \ldots, x_{k}\right)=I^{s}$. So $\left(I^{s}\right)_{k}=I^{s}$ follows from Theorem 2 as required. This completes the proof of Theorem 4 .

Corollary 4(A). Fix the setup of Theorem 4 . If $\mathrm{gr}_{I} R$ is Cohen-Macaulay then $I=I_{d}=\cdots=I_{1}$. In fact $I^{s}=\left(I^{s}\right)_{d}=\cdots=\left(I^{s}\right)_{1}$.

Remark 4(B). Let $R=k\left[\left|x_{1}, \ldots, x_{k}\right|\right]$, where $k$ is a field and $x_{1}, \ldots, x_{d}$ are indeterminates. Let $I=\left(x_{1}^{2}, \ldots, x_{d}^{2}\right) R$. Then $\operatorname{gr}_{I} R$ is Cohen-Macaulay. Thus $I=I_{d}=\cdots=I_{1} \neq \bar{I}$. In fact, $I^{s}=\left(I^{s}\right)_{d}=\cdots=\left(I^{s}\right)_{1} \neq \bar{I}^{s}$. 
Remark 4(C). Craig Huneke showed me (using a PC) the following example: Let $R=k[|x, y|]$ with $k$ a field and $x, y$ indeterminates. Let $I=$ $\left(x^{6}, x^{3} y^{3}, x^{2} y^{4}, y^{6}\right) R$. Then $I_{2} \neq I_{1}$.

Lemma 5(A) (Heisuke Hironaka [Hi]). Let $R$ be a Noetherian ring with $I$ an ideal in $R$. Let $x \in I^{t}-I^{t+1}$. Let $x^{\prime}$ be the image of $x$ in $I^{t} / I^{t+1}$. Suppose $x^{\prime}$ is a regular element in $\operatorname{gr}_{I} R$. Set $R^{\prime}=R /(x)$ and $I^{\prime}=(I, x) /(x)$. Then $\operatorname{gr}_{I} R /\left(x^{\prime}\right) \approx \mathrm{gr}_{I^{\prime}} R^{\prime}$.

Proof. $\mathrm{gr}_{I} R / K \approx \mathrm{gr}_{i^{\prime}} R^{\prime}$ follows from [4, Lemma 5] where $K=\bigoplus_{0}^{\infty} K_{n}$, where $K=\left((x) \cap I^{n}+I^{n+1}\right) / I^{n+1}$. Now if $n \leq t$ then $K_{n}=I^{n+1} / I^{n+1}$. On the other hand, if $n \geq t$ then $K_{n}=\left(x I^{n-t}+I^{n+1}\right) / I^{n+1}$. This follows from the fact that $x^{\prime}$ is a regular element in $\operatorname{gr}_{I} R$, giving $(x) \cap I^{n}=x I^{n-t}$ for $n \geq t$. So $K=\left(x^{\prime}\right)$, proving the lemma.

Lemma 5(B). Let $R$ be a local Noetherian ring with maximal ideal $M$. Let $I$ be an $M$-primary ideal. Set $T_{s}=\operatorname{gr}_{I^{s}} R$ for $s \geq 1$. Set $N_{s}=$ maximal irrelevant homogeneous ideal of $T_{s}$. Then grade $N_{1} \geq k$ implies grade $N_{s} \geq k$.

Proof. Fix $T_{s}$. Assume $k=1$. Then no associated prime of $T_{1}$ can equal $N_{1}$. So there is an element $x \in I^{s m}-I^{s m+1}$ such that $x^{\prime}$ (= image of $x$ in $I^{s m} / I^{s m+1}$ ) is a regular element in $T_{1}$. We claim that $x^{\prime \prime}$ (= image of $x$ in $\left.I^{s m} / I^{s m+s}\right)$ is a regular element in $T_{s}$. Suppose $y \in I^{s n}-I^{s n+s}$ with $x y \in I^{s m+s n+s}$. We may also suppose $y \in I^{s n+i}-I^{s n+i+1}$ for some $i$ with $0 \leq i \leq s$. Then $x y \in I^{s m+s n+i+1}$. By taking order images of $x, y$ in $T_{1}$ we obtain a contradiction. This shows that $x^{\prime \prime}$ is a regular element in $T_{s}$. So lemma holds for $k=1$.

Now set $N_{s}^{\prime}=$ maximal irrelevant homogeneous ideal of $\operatorname{gr}_{L^{\prime}} R^{\prime}$ where $R^{\prime}=$ $R /(x)$ and $L^{\prime}=\left(I^{s}, x\right) /(x)$. Assume grade $N_{1} \geq k>1$. Then grade $N_{1}^{\prime} \geq$ $k-1 \geq 1$ follows from Lemma 5(A). We may assume by induction on dimension that grade $N_{s}^{\prime} \geq k-1$. It follows that grade $N_{s} \geq k$. This completes the proof of the lemma.

Corollary 5(C) (Grothe-Hermann-Orbanz [GHO, Theorem 4.7]). Let $R$ be a local ring with maximal ideal $M$. Let $I$ be an $M$-primary ideal. Then $\operatorname{gr}_{I} R$ is Cohen-Macaulay implies $\operatorname{gr}_{I^{s}} R$ is Cohen-Macaulay for all $s \geq 1$.

Proof of Theorem 5. Fix $s$. Let $x_{i}^{\prime}$ denote the image of $x_{i}$ in $\left(I^{S}\right)^{N} /\left(I^{S}\right)^{N+1}$. We claim that $\operatorname{grade}\left(x_{1}^{\prime}, \ldots, x_{j}^{\prime}\right) T_{s} \geq \operatorname{grade} N_{s}-(d-j)$, where notation is as in Lemma 5(B) and Theorem 2. Suppose the contrary. Then $\operatorname{grade}\left(x_{1}^{\prime}, \ldots, x_{j}^{\prime}\right) T_{s}$ $<\left[\operatorname{grade} N_{s}-(d-j)\right] \operatorname{gives} \operatorname{grade}\left(x_{1}^{\prime}, \ldots, x_{j}^{\prime}, \ldots, x_{d}^{\prime}\right) T_{s}<\left[\operatorname{grade} N_{s}-\right.$ $(d-j)]+(d-j)$. So $\operatorname{grade}\left(x_{1}^{\prime}, \ldots, x_{d}^{\prime}\right) T_{s}<\operatorname{grade} N_{s}$, which is a contradiction. This establishes the claim. 
Now grade $N_{1} \geq k$ implies grade $N_{s} \geq k$. This gives grade $\left(x_{1}^{\prime}, \ldots, x_{j}^{\prime}\right) T_{s} \geq$ $k-(d-j) \geq 1$. So $\left(0^{\prime}: x_{1}^{\prime}, \ldots, x_{j}^{\prime}\right) T_{s}=0^{\prime}$ in $T_{s}$. Consequently

$$
\left(\left(I^{s}\right)^{N+1}:\left(x_{1}, \ldots, x_{j}\right)\right)=I^{s} \text { for every } N \geq 0 \text {. }
$$

So $\left(I^{s}\right)_{j}=I^{s}$ follows from Theorem 2. This completes the proof of Theorem 5 .

\section{ACKNOWLEDGMENTS}

It is a pleasure to thank Craig Huneke for his help, guidance and encouragement. It is a pleasure to thank Jack Ratliff and Dave Rush for all their help.

\section{REFERENCES}

[F] R. Froberg, Connections between a local ring and its associated graded ring, J. Algebra 111 (1970), 333-340.

[Fu] W. Fulton, Introduction to intersection theory in algebraic geometry, CBMS Regional Conf. Ser. in Math., no. 54, Amer. Math. Soc., Providence, R.I., 1983.

[GHO] U. Grothe, M. Hermann, and U. Orbanz, Graded Cohen-Macaulay rings associated to equimultiple ideals, Math. Z. 186 (1986), 531-556.

[Hi] H. Hironaka, Resolution of singularities of an algebraic variety over a field of characteristic 0, Ann. of Math. 79 (1964), 109-326.

[Ho] M. Hochster, Constraints on systems of parameters, Proc. Oklahoma Ring Theory Conf., Dekker, New York, 1974, pp. 121-161.

[HH] M. Hochster and C. Huneke, Tightly closed ideals, Bull. Amer. Math. Soc. (N.S.) 18 (1988), 45-48.

[Hu] C. Huneke, Hilbert functions and symbolic powers, Michigan Math. J. 34 (1987), 293-318.

[Li1] J. Lipman, Equimultiplicity, reduction, and blowing up, Lecture Notes in Pure and Appl. Math., Vol. 68, Dekker, 1982.

[Li2] _ Relative Lipschitz-saturation, Amer. J. Math. 97 (1975), 791-813.

[Ma] H. Matsumura, Commutative algebra, Benjamin, New York, 1970.

[Mc] S. McAdam, Asymptotic prime divisors, Lecture Notes in Math., Vol. 1023, Springer-Verlag, 1983.

[NR] D. G. Northcott and D. Rees, Reductions of ideals in local rings, Proc. Cambridge Philos. Soc. 50 (1954), 145-158.

[N] D. G. Northcott, Lessons on rings, modules and multiplicities, Cambridge Univ. Press, 1968.

[Ra] L. J. Ratliff, On quasi-unmixed local domains, the altitude formula, and the chain condition for prime ideals. II, Amer. J. Math. 92 (1970), 99-144.

[RR] L. J. Ratliff and D. Rush, Two notes on reductions of ideals, Indiana Univ. Math. J. 27 (1978), 929-934.

[R1] D. Rees, $\alpha$-transforms of local rings and a theorem on multiplicities of ideals, Cambridge Philos. Soc. 57 (1961), 8-17.

[R2] - Lectures on the asymptotic theory of ideals, London Math. Soc. Lecture Note Ser., Vol. 113, Cambridge Univ. Press, 1988.

[Sa] P. Samuel, La notion de multiplicité en algèbre et en géométrie algébrique, J. Math. Pures Appl. 30 (1951), 159-274.

[S] K. Shah, Coefficient ideals of the Hilbert polynomial and integral closures of parameter ideals, Ph.D. thesis, Purdue Univ., 1988.

Department of Mathematics and Computer Science, University of Calfornia, RiverSIDE, CALIFORNIA 92521 